\title{
GMR
}

\section{Development of expressed sequence tag-based microsatellite markers for the critically endangered Isoëtes sinensis (Isoetaceae) based on transcriptome analysis}

\author{
A.W. Gichira ${ }^{1,2}$, Z.C. Long, ${ }^{1,2}$, Q.F. Wang ${ }^{1}$, J.M. Chen ${ }^{1}$ and K. Liao ${ }^{1}$ \\ ${ }^{1}$ Key Laboratory of Plant Germplasm Enhancement and Specialty Agriculture, \\ Wuhan Botanical Garden, Chinese Academy of Sciences, Wuhan, China \\ ${ }^{2}$ University of Chinese Academy of Sciences, Beijing, China \\ Corresponding author: K. Liao \\ E-mail: liaokuo@wbgcas.cn \\ Genet. Mol. Res. 15 (3): gmr.15038497 \\ Received January 26, 2016 \\ Accepted March 21, 2016 \\ Published July 15, 2016 \\ DOI http://dx.doi.org/10.4238/gmr.15038497
}

Copyright (C) 2016 The Authors. This is an open-access article distributed under the terms of the Creative Commons Attribution ShareAlike (CC BY-SA) 4.0 License.

\begin{abstract}
Isoëtes sinensis is a critically endangered quillwort. To facilitate studies on the conservation genetics of this species, we developed expressed sequence tag-simple sequence repeat (EST-SSR) markers. A total of 50,063 unigenes were predicted by transcriptome sequencing, 5294 (10.6\%) of which significantly matched 3011 Gene Ontology annotations and 2363 were assigned to Kyoto Encyclopedia of Genes and Genomes metabolic pathways. Most of these (2297) were involved in metabolism. A total of 1982 SSR motifs were identified, with trinucleotides being the dominant repeat motif, and 1438 (72.6\%) SSR primers were designed. Eighteen randomly selected primer pairs were used to genotype 24 I. sinensis accessions, which confirmed the suitability of these novel markers for molecular studies of $I$. sinensis.
\end{abstract}


The heterozygosity index value ranged between 0.0799 and 0.9106 , while the Shannon-Wiener diversity index value ranged between 0.1732 and 2.5589. The EST-SSRs reported in this study are linked to genic sequences, and are therefore ideal for investigating the evolutionary history of I. sinensis. These markers, together with the large EST dataset generated in this study, will greatly facilitate conservation genetic studies of $I$. sinensis.

Key words: De novo assembly; EST-SSR; Isoëtes sinensis; Microsatellite; RNA-sequencing; Transcriptome

\section{INTRODUCTION}

Isoëtes sinensis Palmer is a heterosporous, tetraploid aquatic quillwort that is endemic to East Asia (Liu et al., 2002; He et al., 2004). In China, I. sinensis is found in freshwater intertidal zones, valley marshes and abandoned fields in the southeast region. It often grows close to farmland, and consequently is subject to intense competition from other hydrophyte species because of increased fertilizer input (Pang et al., 2003). Recently, I. sinensis populations have declined steadily (Kang et al., 2005; Liu et al., 2005; Zhu, 2006), and only two populations remain in China (Chen et al., 2012).

I. sinensis is now considered rare and critically endangered, and is listed in the first category of the national key that protects wild plants (Yu, 1999; Liu et al., 2005) and in the International Union for Conservation of Nature (IUCN) Red List (IUCN, 2008). It is an evolutionarily relevant species, because it belongs to a genus that has been in existence since the Devonian geological period, and phylogenetic studies have classified it among basal vascular plants (Pigg, 2001). I. sinensis provides environmental services such as air purification, and is a good bioremediation agent. The continuous reduction of its population has been associated with water quality deterioration in regions where it has ceased to grow (Wen et al., 2003). Therefore, appropriate means of conservation should be formulated to save this species from extinction, and to achieve this, proper molecular analysis is essential.

Consequently, in recent years, genetic variation in I. sinensis has been studied using a variety of genetic markers, e.g., allozymes (Chen et al., 2009), random-amplified polymorphic DNA (Chen et al., 2004), inter-simple sequence repeats (Chen and Wang, 2006), amplified fragment length polymorphisms (AFLPs) (Kang et al., 2005), and simple sequence repeats (SSRs) (Chen et al., 2012). However, the results obtained by these studies do not adequately elucidate the effects of genetic variation and natural selection on populations of I. sinensis. For example, no molecular markers that are linked to specific functional genes, such as expressed sequence tag (EST)-SSRs, have been developed for this critically endangered species. EST-SSRs, also referred to as genic simple sequence repeats, have gained popularity in plant molecular research due to their efficacy in evaluating functional diversity in natural populations. They are obtained from transcribed regions of DNA, and are used in dataset homology searches in order to ascertain their putative functions (Varshney et al., 2005).

The main objectives of our study were as follows: 1) to construct a transcriptome dataset in order to facilitate the discovery of novel genes and to study the comparative genomics of I. sinensis; 2) to develop EST-SSR markers using high-throughput RNA sequencing (RNA-Seq); and 3) to evaluate the viability of the EST-SSR markers developed by testing randomly selected primers.

Genetics and Molecular Research 15 (3): gmr.15038497 


\section{MATERIAL AND METHODS}

\section{RNA extraction}

Total RNA was extracted from leaf tissues of I. sinensis. Samples were collected from four accessions cultivated at Wuhan Botanical Garden, and were immediately frozen in liquid nitrogen. One milliliter of TRIzol reagent (Invitrogen, CA, USA) was added for every 100 $\mathrm{mg}$ leaf tissue and treated with RNase-free DNase 1 (TaKaRa Bio, Shandong, China) for $1 \mathrm{~h}$ at $37^{\circ} \mathrm{C}$. RNA was then dissolved in RNase-free water (Ambion, USA). A $1-\mu \mathrm{L}$ aliquot of each sample was used to check RNA quality and concentration with NanoDrop 2000 Spectrophotometer (Thermo Fischer Scientific Inc., DE, USA) and Agilent Bioanalyzer 2100 (Agilent Technologies, Santa Clara, CA, USA). To maximize the quality of the transcriptional units and improve the analytical process, a pool was created by mixing equal volumes of total RNA from each sample.

\section{cDNA synthesis and sequencing}

A working concentration of $50 \mathrm{ng} / \mu \mathrm{L}$ of total RNA was used for cDNA synthesis. mRNA was isolated from the total RNA pool and purified using a MicroPoly(A)Purist ${ }^{\mathrm{TM}}$ mRNA Purification Kit $\left(\right.$ Ambion $\left.^{\mathrm{TM}}\right)$ following the manufacturer's protocol. cDNA was then synthesized from the mRNA fragments according to the protocol developed by Ng et al. (2005), with slight modifications. First-strand cDNA was synthesized from $10 \mu \mathrm{g}$ mRNA templates using Gsu1-oligo(dT) and $1000 \mathrm{U}_{\text {SuperScript }}{ }^{\mathrm{TM}}$ II Reverse Transcriptase (Invitrogen). The mixture was incubated at $42^{\circ} \mathrm{C}$ for $1 \mathrm{~h}$. The cDNA was then treated with sodium periodate (Sigma, USA) and mRNA 5' oxide caps were biotinylated and connected using Dynabeads ${ }^{\mathrm{TM}}$ M-280 beads (Invitrogen). The alkaline lysis method was used to release first-strand cDNA from the biotin-linked mRNA/cDNA. Second-strand cDNA was synthesized using Ex Taq ${ }^{\mathrm{TM}}$ Polymerase (TaKaRa, Japan), and the GsuI restriction enzyme was used to cut-off poly(A) tails. Double-stranded cDNA was purified using a QIAquick ${ }^{\circledR}$ Polymerase Chain Reaction (PCR) Extraction Kit (Qiagen, Hilden, Germany), and the sequencing adaptors were ligated onto the fragments. Fragments whose sizes were between 300 and 500 bp were selected and purified using AMPure ${ }^{\circledR}$ beads (Agencourt, USA), and then enriched by PCR to construct a library for transcriptome sequencing and to improve the quality of the sequencing process by ensuring fragment uniformity. Finally, the library was sequenced using an Illumina $\mathrm{HiSeq}^{\mathrm{TM}}$ 2000 platform (Illumina Inc., USA).

\section{De novo assembly and functional annotation of unigenes}

Prior to assembly, stringent filtering was conducted, and only high-quality reads were assembled using the Trinity software (Grabherr et al., 2011). This was followed by the prediction and identification of putative open reading frames and untranslated regions within the unigenes using the EMBOSS software (Rice et al., 2000). The predicted proteincoding sequences were compared using the Swiss-Prot and TrEMBL protein databases, with a BLASTp alignment and an E-value set at less than 1E-5.

We used the Perl-based GoPipe program (Chen et al., 2005) to assign Gene Ontology (GO) annotations; in BLASTp, the E-value was again set at less than 1E-5. Metabolic pathway

Genetics and Molecular Research 15 (3): gmr.15038497 
construction was conducted using the Kyoto Encyclopedia of Genes and Genomes (KEGG) database (Kanehisa et al., 2010). The E-value for a bi-directional BLAST was also set at less than 1E-5. The unigenes were assigned KEGG Orthology numbers and their involvement in the metabolic pathway was ascertained.

\section{SSR mining, primer design, and marker validation}

SSR motifs were detected using the program MiSa (http://pgrc.ipk-gatersleben.de/ misa/); mononucleotides were excluded from the search. We used the Primer 3.0 software (Rozen and Skaletsky, 2000) to design the SSR primers. I. sinensis samples were collected from 24 individuals from natural populations in Zhejiang Province, China. About $5 \mathrm{~g}$ fresh leaves was harvested from each plant and immediately dried in a Zip lock plastic bag containing $70 \mathrm{~g}$ silica gel. Samples were stored at room temperature until the DNA was isolated in the laboratory. Total genomic DNA was extracted from each individual using a MagicMag Genomic DNA Micro Kit (Sangon Biotech Co., Shanghai, China) following the manufacturer protocol. DNA quality and concentration were checked by $1.5 \%$ gel electrophoresis with a NanoDrop (Thermo Scientific Inc.). Firstly, we randomly selected 25 SSR primers and tested them for amplification and specificity, from which we selected the 18 best primers and labeled the forward sequence with an FAM fluorescent dye at the 5'-end. The 25- $\mu \mathrm{L}$ PCR mixture contained $2 \mu \mathrm{L} 50-100 \mathrm{ng}$ genomic DNA, $2.5 \mu \mathrm{L}$ 10X Taq buffer (plus $\mathrm{Mg}^{2+}$ ), $0.25 \mathrm{mM}$ of each dNTP, $0.25 \mu \mathrm{M}$ of each primer, 0.2 U Taq polymerase (TaKaRa Bio, Dalian, China), and PCR water.

PCR amplification was conducted using T100 ${ }^{\mathrm{TM}}$ Thermal Cycler (Bio-Rad, CA, USA) with the following cycling parameters: an initial denaturation step of $3 \mathrm{~min}$ at $95^{\circ} \mathrm{C}$, followed by 30 cycles of denaturation for $30 \mathrm{~s}$ at $95^{\circ} \mathrm{C}$, annealing for $30 \mathrm{~s}$ at $50-55^{\circ} \mathrm{C}$, an extension of $30 \mathrm{~s}$ at $72^{\circ} \mathrm{C}$, and a final extension step for $10 \mathrm{~min}$ at $72^{\circ} \mathrm{C}$. The quality of the PCR products was checked on $1.5 \%$ agarose gel prior to sequencing, and the sequences were analyzed using the GeneMarker ${ }^{\circledR}$ software (SoftGenetics). The polymorphic data recorded were used to estimate the intrapopulation genetic diversity of I. sinensis using the ATETRA 1.2 program (Van Puyvelde et al., 2010).

\section{RESULTS}

\section{Illumina de novo sequencing and assembly}

In order to investigate the $I$. sinensis transcriptome, RNA was extracted from several different tissues and a library was successfully created and sequenced using Illumina pairedend technology. A total of 19,522,164 reads with a length of $100 \mathrm{bp}$ each were generated. The reads were assembled into 125,629 contigs that were reduced to 50,110 after a strict filtration step that removed adaptors and low-quality sequences. The average length of the high-quality contigs was $755 \mathrm{bp}$, and ranged between 201 and 14,750 bp. Further processing was conducted on the 50,110 contigs using the EMBOSS getorf tool (Rice et al., 2000) to generate 50,063 unigenes.

\section{Functional annotation and classification}

A GO-based functional annotation of the ESTs was performed through BLASTp using

Genetics and Molecular Research 15 (3): gmr.15038497 
the National Center for Biotechnology Information database. The BLAST results revealed that of the 50,063 unigenes generated, $9979(19.9 \%)$ were homologous to known proteins and $40,084(80.1 \%)$ were homologous to expressed, hypothetical, or unknown proteins. Further analyses conducted using the GoPipe program revealed that $5294(10.6 \%)$ of the unigenes significantly matched 3011 GO functional terms (Figure 1). These unigenes were grouped into three main categories of functional classification: cellular components, biological processes, and molecular functions. A single unigene could have been linked to one or several cellular components, active in one or more biological processes, and involved in one or more molecular functions; therefore, some unigenes were simultaneously annotated into two or three categories. Figure 1 shows that assignments to biological processes constituted the majority $(17,039,37.3 \%)$, followed by molecular functions $(14,641,32.1 \%)$ and cellular components $(13,944,30.6 \%)$.

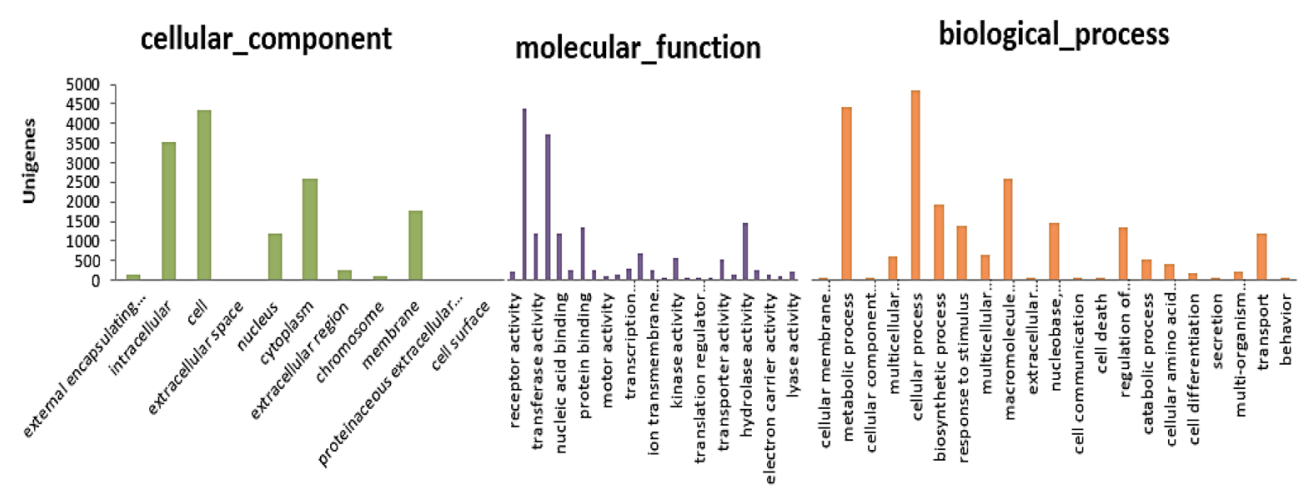

Figure 1. Gene Ontology (GO) classification of 3011 unigenes.

A search of the KEGG database revealed that of the 50,063 unigenes identified, $2363(4.73 \%)$ were involved in KEGG pathways and were assigned to five main categories (Figure 2). The categories were metabolism, general information processing, environmental information processing, cellular processes, and organismal systems. These classifications were further reduced to $25 \mathrm{KEGG}$ pathways, most of which were in line with the biological processes already observed in the GO annotations, including some unigenes being annotated into two or more classifications.

\section{EST-SSR marker development and characterization}

MiSa was employed to further analyze the 50,063 unigenes generated; using the default parameters, 1982 SSR motifs were discovered. We then analyzed the frequency of different repeat motif types (Table 1). The repeat motifs were not equally represented; trinucleotides had the highest percentage (61.3\%), dinucleotides accounted for $25.3 \%$, and tetranucleotides accounted for $10.9 \%$. Compound repeats $(3.7 \%)$ and penta-hexanucleotides $(2.5 \%)$ were the least frequent, and the most common di-, tri-, and tetranucleotide motifs were TA/TA (18.2\%), GGA/TCC (8.1\%), and CTTC/GAAG (8.7\%), respectively (Figure 3). 


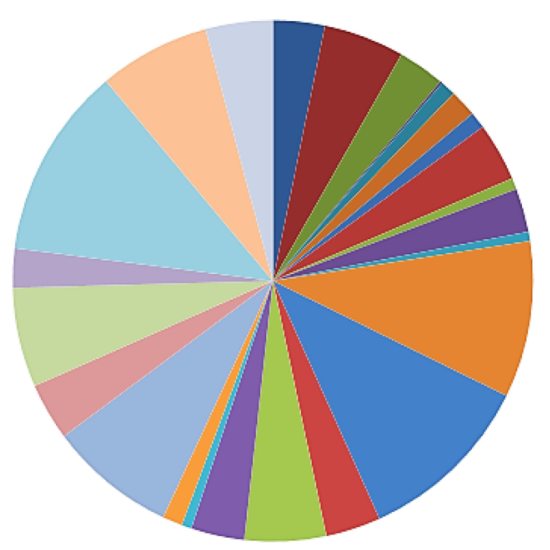

- Amino acid transport and metabolism - Carbohydrate transport and metabolism - Cell cycle control,cell division,chromosome partitioning a Cell motility

- Cell wall/membrane/envelope biogenesis

a Chromatin structure and dynamics

Coenzyme transport and metabolism

$\square$ Coenzyme transp
a cytoskeleton

$\square$ Defense mechanisms

- Energy production and conversion

a Extracellular structures

- Function unknown

- General function prediction only

- Inorganic ion transport and metabolism

- Intracellular trafficking,secretion,and vesicular transport

- Lipid transport and metabolism

- Nuclear structure

- Nucleotide transport and metabolism

- Posttranslational modification, protein turnover, chaperones

a Replication,recombination and repair

$\square$ RNA processing and modification

- Secondary metabolites biosynthe sis, transport and catabolism

- Signal transduction mechanisms

- Transcription

$\square$ Translation,ribosomal structure and biogenesis

Figure 2. Kyoto Encyclopedia of Genes and Genome (KEGG) assignment of 2363 unigenes based on cellular processes, genetic information processing, environmental information processing, metabolism, and organismal systems.

Table 1. Distribution of expressed sequence tag-simple sequence repeat motifs based on the number of repeat units.

\begin{tabular}{l|c|c|c|c|c|c}
\hline Repeats & Di- & Tri- & Tetra- & Penta-Hexa & Total & Percentage \\
\hline 5 & 0 & 676 & 157 & 14 & 847 & 44.4 \\
\hline 6 & 265 & 237 & 26 & 5 & 533 & 27.9 \\
\hline 7 & 130 & 241 & 11 & 2 & 384 & 20.1 \\
\hline 8 & 33 & 16 & 8 & 20 & 77 & 4.0 \\
\hline 9 & 11 & 1 & 0 & 2 & 14 & 0.7 \\
\hline 10 & 13 & 0 & 0 & 4 & 17 & 0.9 \\
\hline 11 & 25 & 0 & 2 & 0 & 27 & 1.4 \\
\hline$\geq 12$ & 6 & 0 & 4 & 0 & 10 & 0.5 \\
\hline Total & 483 & 1171 & 208 & 47 & 1909 & 100.0 \\
\hline Percentage & 25.3 & 61.3 & 10.9 & 2.5 & 100.0 & \\
\hline
\end{tabular}

Di- $=$ dinucleotides; Tri- $=$ trinucleotides; Tetra- $=$ tetranucleotides; Penta-Hexa $=$ pentanucleotides and hexanucleotides.

\section{Validation of EST-SSR primers}

SSR primers were designed using the Primer 3.0 program based on the flanking regions of the SSR-containing sequences. A total of 1438 SSR primers (72.6\%) were designed. Twenty-five primer pairs were randomly selected and tested for amplification and specificity using genomic DNA extracted from four accessions of I. sinensis. Eighteen (72\%) markers were successfully amplified, produced clear bands after gel electrophoresis, and generated products of the expected size at various annealing temperatures.

We genotyped 24 accessions in order to authenticate the markers and their suitability for further molecular studies of I. sinensis. All 18 markers exhibited a high level of polymorphism and a significant amount of diversity was observed. Heterozygosity values ranged from 0.08 to 0.91 , and Shannon-Wiener diversity index values ranged from 0.17 to 2.55 . The locus sequences have been submitted to GenBank (Table 2).

Genetics and Molecular Research 15 (3): gmr.15038497 


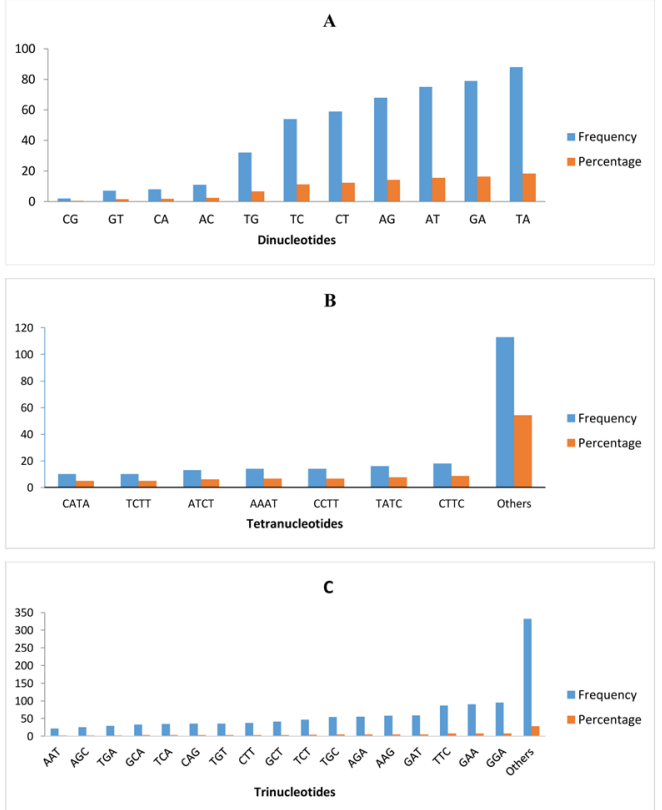

Figure 3. Frequency distribution (number and percentage) of the main expressed sequence tag-simple sequence repeats based on motif type. A. Dinucleotides. B. Tetranucleotides. C. Trinucleotides.

\begin{tabular}{|c|c|c|c|c|c|c|c|}
\hline Locus & Repeat motif & \begin{tabular}{|l} 
Primer 5'-3' \\
\end{tabular} & GenBank accession No. & $\operatorname{Tm}\left({ }^{\circ} \mathrm{C}\right)$ & Allele size range (bp) & $\mathrm{H}_{\mathrm{i}}$ & $\mathrm{H}^{\prime}$ \\
\hline SJSSR4 & $(\mathrm{GCA})_{5} \mathrm{~N}(\mathrm{CAG})_{5}$ & $\begin{array}{l}\text { F:GCAGCAGATTCAGATGCAGA } \\
\text { R: GTTCCTGATGCTGGTGGTCT }\end{array}$ & KT989976 & 53 & $145-198$ & 0.737 & 1.5301 \\
\hline SISSR5 & $(\mathrm{CA})_{6}$ & $\begin{array}{l}\text { F: CACACCCAACATACATACGCA } \\
\text { R: ATGGGGTGAAACAACAGGAG }\end{array}$ & KT989977 & 53 & $144-172$ & 0.8367 & 1.9181 \\
\hline SJSSR6 & $(\mathrm{ACTC})_{6}$ & $\begin{array}{l}\text { F: AGAAAAATGATCGCCTGTGG } \\
\text { R: TGCACTATGGAAAGCTTGTGA }\end{array}$ & KT989978 & 55 & $129-141$ & 0.8307 & 1.9513 \\
\hline SJSSR9 & $\left(\mathrm{TGC}_{6}\right.$ & $\begin{array}{l}\text { F: GCAAAGTCCACCAAGCAGAT } \\
\text { R: AATGCCCTGGCAGTTAACAC }\end{array}$ & KT989979 & 55 & $225-229$ & 0.2176 & 0.3744 \\
\hline SISSR11 & $(\mathrm{GCT})_{6}$ & $\begin{array}{l}\text { F: CAGCATCAATGAGCAAAGGA } \\
\text { R: GCTTGGGGCAAGTAGCTATG }\end{array}$ & KT989980 & 53 & $218-233$ & 0.7688 & 1.5746 \\
\hline SJSSR 12 & $(\mathrm{GAT})_{5}$ & $\begin{array}{l}\text { F: GTGGTTGATTTGGGGTCATC } \\
\text { R: CCCTCTTTGCCAACAGTGAT }\end{array}$ & KT989981 & 53 & $205-240$ & 0.7673 & 1.7722 \\
\hline SJSSR 14 & $(\mathrm{CT})_{10}$ & $\begin{array}{l}\text { F: GGCCAGAGAACAGGAGAAAG } \\
\text { R: CCAAGTGGAAATTATGTCGCT }\end{array}$ & KT989982 & 55 & $255-281$ & 0.8774 & 2.281 \\
\hline SJSSR45 & $(\mathrm{TC})_{6}$ & $\begin{array}{l}\text { F: AAGGCCAACACAAAAACTGG } \\
\text { R: CGCCCACTAATCAGGACACT }\end{array}$ & KT989983 & 52 & $243-255$ & 0.7315 & 1.4196 \\
\hline SJSSR46 & $(\mathrm{AAC})_{7}$ & $\begin{array}{l}\text { F: TCGAAAATTCGAAGATCGCT } \\
\text { R: ATAATTTGGAGGTTGCGACG }\end{array}$ & KT989984 & 52 & $226-252$ & 0.7679 & 1.5833 \\
\hline SJSSR50 & $(\mathrm{TCA})_{5}$ & $\begin{array}{l}\text { F: ACCATAGTGGGGTGAAGGAA } \\
\text { R: CCTGAGTCTCATGTGCAAGC }\end{array}$ & KT989985 & 54 & 195 & 0.0799 & 0.1732 \\
\hline SJSSR53 & $(\mathrm{TCT})_{5}$ & $\begin{array}{l}\text { F: AGGGATTGCTAGCGCTGTTA } \\
\text { R:GGCAAAACAAAAGCATCCAT }\end{array}$ & KT989986 & 54 & $195-286$ & 0.9106 & 2.5589 \\
\hline SJSSR 15 & (TGT)6 6 & $\begin{array}{l}\text { F: CTCTTGCCTCTGCTCTTGCT } \\
\text { R: TGATAGAGGGTGGGAAATCG }\end{array}$ & KT989987 & 55 & $241-275$ & 0.8127 & 1.7587 \\
\hline SJSSR24 & $(\mathrm{TCA})_{6}$ & $\begin{array}{l}\text { F: GTTTGGGCATTTTTCCCTCT } \\
\text { R: TGAAAAGCCTATGGTTGGGT }\end{array}$ & KT989988 & 53 & $131-241$ & 0.7869 & 2.0675 \\
\hline SJSSR32 & (TTCC)s & $\begin{array}{l}\text { F: AAATGCCACCAATCCATCAT } \\
\text { R: AAATCAGGTCCGATGGTCTG }\end{array}$ & KT989989 & 52 & $228-243$ & 0.852 & 1.9835 \\
\hline SJSSR39 & $(\mathrm{GCA})_{6}$ & $\begin{array}{l}\text { F: CAGTGACATGCCCGTTCATA } \\
\text { R: GTGGAGTTGGAGGCAACAAT }\end{array}$ & KT989990 & 53 & 228 & 0.2778 & 0.4506 \\
\hline SJSSR8 & $(\mathrm{GAC})_{7}$ & $\begin{array}{l}\text { F: CTTGGTAGCGAACATGAGCA } \\
\text { R: ATGATGGCATCTTCCTCAGC }\end{array}$ & KT989991 & 53 & $193-206$ & 0.818 & 1.9951 \\
\hline SISSR22 & $(\mathrm{AAC})_{5}$ & $\begin{array}{l}\text { F: TACGCAGAGCCCCTTCTCTA } \\
\text { R: GCCCTCGGTATTGAATCTGT }\end{array}$ & KT989992 & 55 & $197-224$ & 0.5256 & 1.1476 \\
\hline SISSR26 & $($ GTT) 5 & $\begin{array}{l}\text { F: ATAACAAAAAGGGCGCTTGA } \\
\text { R: CTTAATTTCTGCAGGAGCCG }\end{array}$ & KT989993 & 52 & $116-274$ & 0.631 & 1.1042 \\
\hline
\end{tabular}

$\mathrm{Tm}=$ melting temperature; $\mathrm{H}_{\mathrm{i}}=$ heterozygosity index; $\mathrm{H}^{\prime}=$ observed Shannon-Wiener information index.

Genetics and Molecular Research 15 (3): gmr.15038497 


\section{DISCUSSION}

Isoëtes sinensis is listed as critically endangered by the IUCN and as possibly extinct in some regions of China, e.g., Anhui and Jiangsu Provinces (China Plant Specialist Group, 2004). Despite a number of studies being conducted on remnant populations of this species that had the primary objective of saving the remaining individuals and re-establishing thriving populations (Kang et al., 2005; Chen et al., 2004, 2009, 2012), natural populations of this species continue to decline. EST-SSR markers have numerous applications in botany and have been used in conservation genetics, marker-assisted selection, and comparative genomics (Varshney et al., 2007). In this study, we used molecular tools (EST-SSRs) to facilitate further molecular studies of I. sinensis. The number of unigenes generated in this study is comparable to that obtained by other studies that have used Illumina sequencing technology (Chen et al., 2015; Zhou et al., 2016).

Our analyses assigned the SSR motifs discovered into three GO categories: i) biological functions, e.g., enzymatic reactions, plant hormonal regulations, and plant responses to stimuli; ii) cellular functions, e.g., genes that putatively regulate cellular functions including cell activity, nucleus activity, and cytoplasm regulation; and iii) molecular functions, e.g., protein binding, motor activity, and nucleic acid activity. Within the biological processes category, cellular process $(3785,22.21 \%)$ and metabolic process $(3461,20.31 \%)$ were highly represented. Binding $(3681,25.14 \%)$ and catalytic activity $(3113,21.26 \%)$ were the largest groups in the molecular function category, while in the cellular component category, cell $(4353,31.22 \%)$ and intracellular $(3514,25.2 \%)$ contained the majority of the unigenes. KEGG is a knowledge base for mapping molecular datasets in "-omics" studies, including transcriptomics (Kanehisa et al., 2010), and 2363 unigenes were assigned KEGG pathways, indicating that they are involved in metabolic activity. Of the $25 \mathrm{KEGG}$ pathways, signal transduction mechanism (2913) and general function prediction (2696) were the most highly represented, followed by metabolism (2297) and genetic information processing (1023).

A total of 1982 EST-SSR motifs were mined for this species. Trinucleotides were the most frequent $(61.3 \%)$, whereas dinucleotides are the most abundant in another aquatic species, Nelumbo nucifera (Zhang et al., 2014). The most frequent types of repeat among the di- and trinucleotides were TA\TA and GGA\TTC, respectively. The EST-SSRs revealed relatively high intrapopulation genetic diversity in I. sinensis $(0.68)$ compared to that recorded in previous studies using AFLP markers [expected heterozygosity $\left(H_{\mathrm{E}}\right)=0.118$, genetic diversity $\left(H_{\mathrm{s}}\right)=0.147$, and Shannon's index $\left.\left(\mathrm{H}^{\prime}\right)=0.192\right]$ (Kang et al., 2005), allozymes $(0.32)$ (Chen et al., 2009), and SSR markers $\left(H_{\mathrm{E}}=0.361\right.$ for a Jiande population and $H_{\mathrm{E}}=0.390$ for a Xiuning population) (Chen et al., 2012). The high level of genetic diversity observed in the small population sizes of $I$. sinensis may be because reductions in these populations have occurred relatively recently (Kang et al., 2005). In addition, the habitats of the few remaining populations have been disturbed, resulting in overly fragmented and disparate subpopulations; consequently, this results in increased genetic diversity due to isolation. The population sample sizes used in this study were relatively small; therefore, it is difficult to draw a firm conclusion concerning genetic variation in I. sinensis. We recommend that extensive field surveys and the collection of an adequate number of samples should be undertaken in order to investigate the genetic diversity of this species and assist in its conservation.

In summary, 18 informative markers were authenticated from 25 that were randomly selected from 1438 markers. Further polymorphic genic SSR markers for I. sinensis await

Genetics and Molecular Research 15 (3): gmr.15038497 
development. EST-SSRs have a higher transferability rate than genomic SSRs, and a lower mutation rate (Varshney et al., 2005); therefore, the novel polymorphic SSR markers developed in this study can be used in evolutionary biology, phylogeography, and comparative genomic studies of species in the genus Isoëtes.

\section{Conflicts of interest}

The authors declare no conflict of interest.

\section{ACKNOWLEDGMENTS}

We thank Lena Li and Josphat Saina for their assistance in the laboratory. Research supported by grants from the National Natural Science Foundation of China (\#31200170 and \#31570220).

\section{REFERENCES}

Chen H, Liu L, Wang L, Wang S, et al. (2015). Development and validation of EST-SSR markers from the transcriptome of Adzuki Bean (Vigna angularis). PLoS One 10: e0131939. http://dx.doi.org/10.1371/journal.pone.0131939

Chen JM and Wang QF (2006). Genetic diversity in the rare and endangered fern Isoëtes orientalis. J. Wuhan Bot. Res. 24: $569-573$.

Chen JM, Wang JY, Liu X, Zhang YW, et al. (2004). RAPD analysis for genetic diversity of Isoëtes sinensis. Biodivers. Sci. 12: 348-353.

Chen YY, Yang W, Li W, Li ZZ, et al. (2009). High allozyme diversity and unidirectional linear migration patterns within a population of tetraploid Isoëtes sinensis, a rare and endangered pteridophyte. Aquat. Bot. 90: 52-58. http://dx.doi. org/10.1016/j.aquabot.2008.05.008

Chen YY, Kong DR, Huang CH, Xu YX, et al. (2012). Microsatellite analysis reveals the genetic structure and gene flow of the aquatic quillwort Isoëtes sinensis, a critically endangered species in China. Aquat. Bot. 96: 52-57. http://dx.doi. org/10.1016/j.aquabot.2011.09.001

Chen ZZ, Xue CH, Zhu S, Zhou FF, et al. (2005). GoPipe: streamlined gene ontology annotation for batch anonymous sequences with statistics. Prog. Biochem. Biophys. 32: 187-191.

China Plant Specialist Group (2004). Isoëtes sinensis. IUCN Red List of Threatened Species. International Union for Conservation of Nature. Accessed March 22, 2016.

Grabherr MG, Haas BJ, Yassour M, Levin JZ, et al. (2011). Full-length transcriptome assembly from RNA-Seq data without a reference genome. Nat. Biotechnol. 29: 644-652. http://dx.doi.org/10.1038/nbt.1883

He ZC, Wang HC, Li JQ, Ye QQ, et al. (2004). Chromosome behavior during meiosis and development of spore mother cells in the Chinese quillwort Isoëtes sinensis T.C. Palmer (Isoetaceae). Am. Fern J. 94: 183-195. http://dx.doi. org/10.1640/0002-8444(2004)094[0183:CBDMAD]2.0.CO;2

International Union for Conservation of Nature (IUCN) (2008). Red List of Threatened species summary statistics. Accessed on March 22, 2016.

Kanehisa M, Goto S, Furumichi M, Tanabe M, et al. (2010). KEGG for representation and analysis of molecular networks involving diseases and drugs. Nucleic Acids Res. 38: D355-D360. http://dx.doi.org/10.1093/nar/gkp896

Kang M, Ye Q and Huang H (2005). Genetic consequence of restricted habitat and population decline in endangered Isoetes sinensis (Isoetaceae). Ann. Bot. 96: 1265-1274. http://dx.doi.org/10.1093/aob/mci277

Liu H, Wang QF and Taylor WC (2005). Isoëtes orientalis (Isoetaceae), a new hexaploid quillwort from China. Novon 15: 164-167.

Liu X, Wang Y, Wang QF and Guo YH (2002). Chromosome numbers of the Chinese Isoëtes and their taxonomical significance. Acta Phytotax. Sin 40: 351-356.

Ng P, Wei CL, Sung WK, Chiu KP, et al. (2005). Gene identification signature (GIS) analysis for transcriptome characterization and genome annotation. Nat. Methods 2: 105-111. http://dx.doi.org/10.1038/nmeth733

Pang XA, Liu X, Liu H, Wu C, et al. (2003). The geographic distribution and habitat of the Isoëtes plants in China. Biodivers. Sci 11: 288-294.

Genetics and Molecular Research 15 (3): gmr.15038497 
Pigg KB (2001). Isoetalean Lycopsid evolution: from the Devonian to the present. Am. Fern J. 91: 99-114. http://dx.doi. org/10.1640/0002-8444(2001)091[0099:ILEFTD]2.0.CO;2

Rice P, Longden I and Bleasby A (2000). EMBOSS: the European molecular biology open software suite. Trends Genet. 16: 276-277. http://dx.doi.org/10.1016/S0168-9525(00)02024-2

Rozen S and Skaletsky H (2000). Primer3 on the WWW for general users and for biologist programmers. Methods Mol. Biol. 132: 365-386.

Van Puyvelde K, Van Geert A and Triest L (2010). atetra, a new software program to analyse tetraploid microsatellite data: comparison with tetra and tetrasat. Mol. Ecol. Resour. 10: 331-334. http://dx.doi.org/10.1111/j.17550998.2009.02748.x

Varshney RK, Graner A and Sorrells ME (2005). Genic microsatellite markers in plants: features and applications. Trends Biotechnol. 23: 48-55. http://dx.doi.org/10.1016/j.tibtech.2004.11.005

Varshney RK, Chabane K, Hendre PS, Aggarwal RK, et al. (2007). Comparative assessment of EST-SSR, EST-SNP and AFLP markers for evaluation of genetic diversity and conservation of genetic resources using wild, cultivated and elite barleys. Plant Sci. 173: 638-649. http://dx.doi.org/10.1016/j.plantsci.2007.08.010

Wen MZ, Pang XA, Wang QF and Taylor WC (2003). Relationship between water chemistry and the distribution of the endangered aquatic quillwort Isoëtes sinensis Palmer in China. J. Freshwat. Ecol. 18: 361-367. http://dx.doi.org/10 $.1080 / 02705060.2003 .9663971$

Yu YF (1999). A milestone of wild plant conservation in China. Plants 5: 3-11.

Zhang W, Tian D, Huang X, Xu Y, et al. (2014). Characterization of flower-bud transcriptome and development of genic SSR markers in Asian lotus (Nelumbo nucifera Gaertn.). PLoS One 9: e112223. http://dx.doi.org/10.1371/journal. pone. 0112223

Zhou Q, Luo D, Ma L, Xie W, et al. (2016). Development and cross-species transferability of EST-SSR markers in Siberian wildrye (Elymus sibiricus L.) using Illumina sequencing. Sci. Rep. 6: 20549. http://dx.doi.org/10.1038/ $\underline{\text { srep20549 }}$

Zhu S (2006). Community characteristics and interspecific association of the Songyang population of Isoëtes sinensis. Biodivers. Sci. 14: 258-264. http://dx.doi.org/10.1360/biodiv.060014 